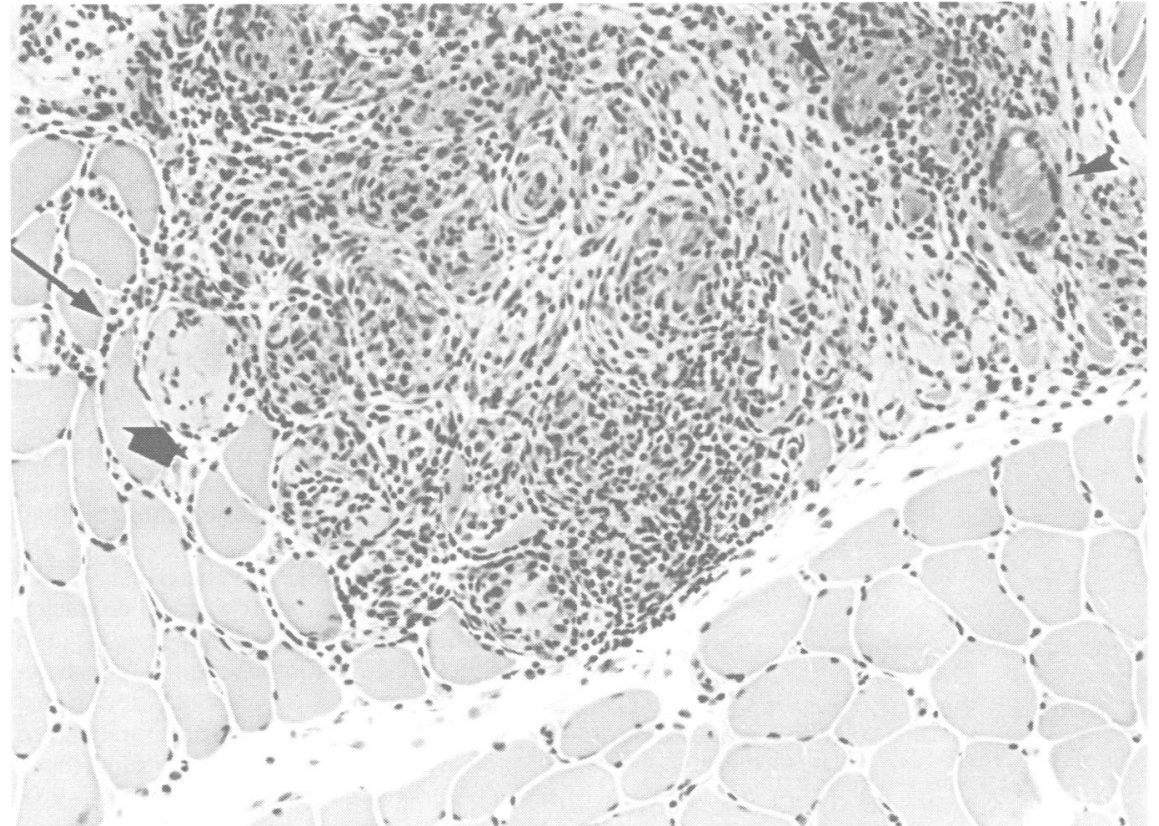

Figure 2 Biopsy of the gastrocnemius muscle showing multiple granulomas in an interfascicular septum. Multinucleated giant cells are present (arrowheads). A lymphocytic infiltrate invades the surrounding muscle fibres (long arrow). A necrotic fibre around the granuloma is shown (thick arrow).

of about $50 \%$ of patients with sarcoidosis, a clinical myopathy is seen in less than $1 \%$ of them, corresponding to $12 \%$ to $26 \%$ of cases of neurosarcoidosis. ' 2 Rarely, a myopathy is the presenting feature: Stern et al found only one such presentation out of 649 patients. ${ }^{1}$ Chapelon et al described three patients with a myopathy as the only clinical manifestation of sarcoidosis during a follow up of 104 months. ${ }^{2}$

Although the course of sarcoid myopathy can be acute or subacute, a chronic course with atrophy (or pseudohypertrophy) seems to be more frequent. It almost always presents as a proximal myopathy, sometimes affecting the bulbar muscles. A distal myopathy, as seen in our patient, is an exceptional presentation of this already unusual disease. Dyken reported one case with proximal weakness in the legs, and with mild distal involvement of the upper limbs although the shoulder girdle muscles were normal. ${ }^{3}$ Chapelon et al reported one patient with a proximal myopathy who also had distal involvement. ${ }^{2}$ Wolfe et al (patient 2) ${ }^{4}$ and Meyer and Regli5 each reported one patient with possible distal involvement, but the coexisting peripheral neuropathy made the interpretation difficult.

The pathogenesis of sarcoid myopathy is uncertain. The inflammation is clearly concentrated in the connective tissue department of the muscle, affecting muscle fibres as "innocent bystanders". Our finding, however, that the infiltrate readily invades muscle fascicles, suggests that a true myositis may contribute to the clinical weakness.

Unfortunately, although inflammation is thought to be a reversible cause of muscle disease and several authors report a favourable effect of steroids on sarcoid myopathy, ${ }^{14}$ we were unsuccessful in treating the patient with steroids. The chronicity of the inflammation accompanied by fibrosis is probably a major factor in this steroid resistance.

In summary, the present case suggests that sarcoidosis should be considered in the differential diagnosis of a distal myopathy and that a chronic myopathy with distal predominance can be the sole manifestation of sarcoidosis for a period as long as four years.

W ROBBERECHT $P$ THEYS

Department of Neurology, University Hospital Gasthuisberg, Leuven, Belgium Department of Pathology, University Hospital Gasthuisberg, Leuven, Belgium
J LEENDERS Department of Neurology, General Hospital St.-Dimpna, Geel, Belgium

1 Stern B, Krumholz A, Johns C, Scott P, Nissim J. Sarcoidosis and its neurological manifestations. Arch Neurol 1985;42:909-17.

Chapelon C, Ziza JM, Piette JC et al. Neurosarcoidosis: signs, course and treatment in 35 confirmed cases. Medicine 1990; 69:261-76.

3 Dyken PR. Sarcoidosis of skeletal muscle. A case report and review of the literature. Neurology 1962;12:643-51.

4 Wolfe SM, Pinals RS, Aelion JA, Goodman RE. Myopathy in sarcoidosis: clinical and pathological study of four cases and review of pathological study of four cases and review of the literat

5 Meyer HJ, Regli F. Über die Beziehungen der Polymyositis granulomatosa Boeck zur late onset myopathy. Disch Z Nervenheik 1965; 186:547-70.

\section{Unilateral proptosis due to cerebellar stroke}

We report a case of unilateral proptosis resulting from a cerebellar stroke causing acute hydrocephalus. Ventriculostomy to alleviate the increased intracranial pressure resulted in regression of the proptosis.

A 73 year old woman was admitted to hospital for right sided hemiparesis that appeared on the morning of her admission. Her medical history included mitral regurgitation, chronic atrial fibrillation, and hypertension. Despite recurrent transient ischaemic attacks in the past she had never received anticoagulant or antiplatelet treatment.
On admission the patient was afebrile, her pulse was 107 beats/min and irregular, her blood pressure was $180 / 100 \mathrm{~mm} \mathrm{Hg}$. An apical systolic murmur was heard. She was lethargic but responded to verbal stimuli. The right pupil was slightly wider than the left, both equally reactive to light. Right sided hemiparesis and an extensor plantar response were noted. Brain CT obtained 48 hours after her admission showed a hypodense lesion within the left cerebellar hemisphere, with surrounding oedema, consistent with a recent stroke in the territory of the left anterior inferior cerebellar artery. On the fourth day in hospital she became somnolent, responding only to painful stimuli. Ptosis and proptosis of the right eye with pronounced chemosis appeared. Oculocephalic response was normal on the left, but she had seventh and partial third nerve palsies on the right. The pupil was dilated and unresponsive to light. Repeated CT showed a large cerebellar infarction with massive oedema, compression of the fourth ventricle, and hydrocephalus (figure). A continuous pressure controlled ventricular drainage system was inserted, with prompt improvement in the patient's alertness and regression of the ocular findings within 24 hours. Her subsequent hospital course was complicated by staphylococcocal septicaemia and she died on the 12th day after her admission.

Unilateral proptosis is almost invariably the result of acute or chronic disorders within or around the orbit. ${ }^{\prime}$ Rarely, it is caused by chronic increase in intracranial pressure. $^{23} \mathrm{We}$ are unaware of a previous report of proptosis as the consequence of a posterior fossa stroke complicated by acute hydrocephalus. Nor has proptosis been associated with other causes of acutely increased intracranial pressure. The postulated mechanism by which intracranial hypertension may lead to exophthalmus is transmission of increased pressure to the orbital veins, resulting in orbital tissue oedema. Proptosis caused by this mechanism is usually symmetric but due to the highly variable drainage of the cavernous sinuses it could be unilateral. ${ }^{+}$

Compression of the fourth ventricle is a recognised complication of cerebellar strokes and surgical interventions may be indicated for relieving hydrocephalus and brain stem

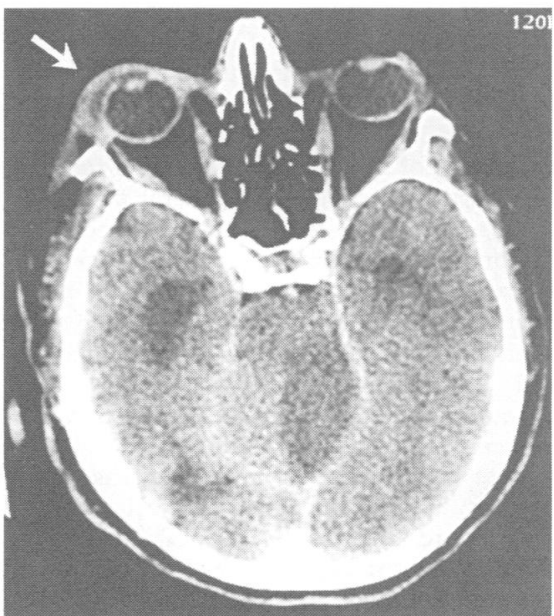

Enhanced axial CT showing hypodensity of the left superior vermis with mass effect compressing the pons and aqueduct. There is secondary enlargement of the temporal horns. The right eye is proptotic with preseptal oedema (arrow). 
pressure. ${ }^{5}$ It should be recognised that in the setting of a cerebellar or brainstem infarction, unilateral proptosis may herald increasing intracranial pressure mandating immediate treatment.

\section{AMIR HALKIN JACOB ABLIN Department of Internal Medicine,
Hadassah Mt Scopus, ferusalem, Israel ISRAEL STEINER Department of Neurology, ferusalem, Israel}

Correspondence to: Dr A Halkin, Department of Internal Medicine, Hadassah University Hospital, Mt Scopus, Jerusalem 91240, Israel.

1 Calcaterra TC, Trapp TK. Unilateral proptosis. Otolaryngol Clin North Am 1988;21: 53-63.

2 Shapiro K, Saiontz H, Shulman K. Unilateral proptosis and visual field defect associated with hydrocephalus [letter]. Arch Neurol 1976;33:663-4.

3 Choudhury AR, Taylor JC, Whitaker R. Unilateral proptosis due to a midbrain tumor. A case report. Acta Ophthalmol (Copenh) 1976;54:762-6.

4 Choudhury AR. Pathogenesis of unilateral proptosis. Acta Ophthalmol (Copenh) 1977; proptosis.

5 Rieke K, Krieger D, Adams HP, Aschoff A, Meyding-Lamade U, Hacke W. Therapeutic strategies in space occupying cerebellar strategies in space occupying cerebellar
infarction based on clinical, neuroradiological and neurophysiological data. Cerebrovascular Diseases 1993;3:45-55.

\section{Normal frontal cortex histology and immunohistochemistry in patients with motor neuron disease}

In the light of the association between frontotemporal dementia and motor neuron disease, ${ }^{12}$ two recent studies have examined neuropsychological function, and distribution of cerebral blood flow $^{3}$ or metabolism, in patients with motor neuron disease without overt clinical evidence of dementia. These authors showed subclinical evidence of the characteristic changes of frontotemporal dementia, with failure on tasks sensitive to frontal lobe function, and hypometabolism, or reduced cerebral blood flow, particularly in the orbitofrontal area.

Brains from patients with motor neuron disease with clinically evident frontotemporal dementia show both microvacuolation of the outer cortical laminae ${ }^{5}$ and ubiquitinated inclusions $^{6}$ in neurons of the superficial layers of the frontal and temporal cortices; inclusions are also seen in hippocampa granule cells. ${ }^{6}$ We have therefore examined the brains of 17 unselected patients referred for routine necropsy to a general pathology department with the clinical diagnosis of motor neuron disease, without dementia. Formal neuropsychological testing had not been carried out. Brains were stained with haematoxylin and eosin for routine histological evaluation, and immunostained for ubiquitin, using standard techniques, for detection of inclusions. Ubiquitinated inclusions typical of motor neuron disease were present in the anterior horn cells of the spinal cord in all cases; frontal cortex was normal, with no evidence of either microvacuolation or inclusions.

Patients with motor neuron disease and frontotemporal dementia usually present with dementia, subsequently developing signs of amyotrophy. It is therefore possible that the neuropathological hallmarks of microvacuolation and inclusions are a relatively late feature. It will be important to examine pathologically brains from patients with motor neuron disease who have been prospectively tested in life; nevertheless others have failed to demonstrate frontal cortical atrophy in motor neuron disease without dementia, ${ }^{7}$ and it is interesting that patients with motor neuron disease can seemingly have significant neuropsychological and functional deficits without overt evidence of structural or pathological changes.

N COOPER alton Centre, Rice Lane,

Liverpool L9 $1 A E, U K$

M SIDDONS

Pathological Sciences, University of Manchester,
Oxford Road,

Manchester M13, UK

Correspondence to: Dr Cooper.

1 Morita $K$, Kaiya $H$, Ikeda $T$, Namba $M$ Presenile dementia combined with amyotrophy: a review of 34 Japanese cases. Arch Gerontol Geriatr 1987;6:263-77.

2 Neary D, Snowden JS, Mann DMA, Northen B, Goulding PJ, Macdermott N. Fronta lobe dementia and motor neurone disease. Neurol Neurosurg Psychiatry 1990;53:23-32.

3 Talbot PR, Goulding PJ, Lloyd JJ, Snowden JS, Neary D, Testa HJ. Inter-relation between "classic" motor neuron disease and fronto-temporal dementia: A neuropsychological and single photon emission computed logical and single photon emission computed tomography study. $\mathcal{F}$

4 Kew JJM, Goldstein LH, Leigh PN, Abrahams S, Cosgrave N, Passingham RE, et al. The
, the relationship between abnormalities of cognitive function and cerebral activation in amyotrophic lateral sclerosis-a neuropsychological and positron emission tomography study. Brain 1993;116:1399-423.

5 Neary D, Snowden JS, Mann DMA. The clinical pathological correlates of lobar atrophy. Dementia 1993;4:154-9.

6 Okamoto K, Murakami N, Kusaka H, Yoshida M, Hashizume Y, Nakazato Y, et al. Ubiquitin-positive intraneuronal inclusion in the extramotor corticies of presenil dementia patients with motor

7 Kiernan JA, Hudson AJ. Frontal lobe atrophy in motor neuron diseases. Brain 1994 117:747-57.

Very severe amnesia with acute onset after isolated hippocampal damage due to systemic lupus erythematosus

Neuropsychiatric lupus designates the whole range of behavioural and cognitive impairments occurring in systemic lupus erythematosus. Up to $75 \%$ of patients with systemic lupus erythematosus may be affected. Typical manifestations are strokes, seizures, dementia, psychosis, and confusional states. ${ }^{1}$ We describe a patient with acute onset of isolated, extremely severe, and persistent global amnesia due to systemic lupus erythematosus.

This $\mathbf{5 5}$ year old previously healthy farmer was confused after a nap in the afternoon He had no known vascular risk factor. A admission, he was alert but confused for time and place and repeatedly asked the same questions about where he was and what had happened to him. Physical examination was normal. He could not repeat three words after a two minute delay. Routine blood tests were normal except for thrombocytopenia $\left(26 \times 10^{\circ} / 1\right)$. His CSF had a normal cell count, protein, and glucose content.

His behaviour was remarkable only for a most pervasive amnesia: the patient never recalled visitors, specifics from test sessions, or daily events. He always recognised his family and long time friends but never the examiners or other people on the ward. $\mathrm{He}$ easily oriented himself in his own home but did not find his way around on the ward, where he spent three months. Whereas very remote memory seemed preserved, he was unaware of events of the past 10 to 15 years. He easily recognised the photographs of cattle he had owned more than 15 years ago, but not those he had owned more recently. He never confabulated but always indicated that he did not know the answers to questions. He was initially placid and unconcerned but became depressed after a month; he often cried and complained about his bad memory.

Neuropsychological evaluations established normal oral and written language, arithmetic skills, praxis, finger gnosis, and right-left discrimination as well as constructive abilities, space exploration, and visual recognition of complex material. Intelligence was low average (Wechsler adult intelligence scale, $\quad \mathrm{IQ}=91 ; \quad \mathrm{VIQ}=89 ; \quad \mathrm{PIQ}=96$ ). Attention and cognitive speed were normal. Executive functions were initially deficient (in particular high rate of perseverations in fluency tasks) but reached the normal range in the course. The main finding was a profound amnesia (table). Although the immediate memory span was normal, he was unable to recall any previously acquired explicit information, independent of the modality of presentation. Both in recall and recognition tasks, he denied any familiarity with the items and had a high rate of false positives in forced choice recognition tasks. An autobiographical interview and a test probing knowledge about Swiss politicians disclosed a temporally graded retrograde amnesia extending 10 to 15 years backwards. Semantic memory was normal, as evidenced by normal naming and verbal fluency. Motor learning in a mirror drawing task was normal.

Brain MRI was performed three times. In the initial MRI 10 days after onset, both hippocampi seemed discretely swollen with blurring of the cortical structure. In the MRIs after three and 10 months, both hippocampi appeared considerably smaller than in the initial scan (figure). No additional lesions were found; in particular, there was no indication of small hemispheric or thalamic vascular lesions.

Transcranial and carotid Doppler ultrasound examination and echocardiography were normal. Thrombocytopenia persisted $\left(15\right.$ to $\left.31 \times 10^{9} / 1\right)$. Haemoglobin and leucocyte count, renal function, and liver enzymes were normal. A bone marrow biopsy showed normal haematopoiesis. Antinuclear (1:400), anti-dsDNA, and anti-ssDNA antibodies were raised. No antibodies were detected against ENA-Sm, phospholipid, thrombocytes (anti-GPIIb/IIIa and antiGPIb/IX), or neurotrophic viruses. No antineuronal antibodies were found (sought after five months when antinuclear antibodies were no longer detectable). Complement factor C4 was slightly decreased $(135 \mathrm{mg} / \mathrm{l})$, C3 was normal. A skin biopsy contained deposits of IgG, IgM, and C3 along the basal membrane. These findings were consistent with a diagnosis of systemic lupus erythematosus according to the 1982 American Rheumatism Association criteria.

The patient was treated with prednisone ( $75 \mathrm{mg}$ daily for 12 weeks), cyclophosphamide (150 mg daily for 10 weeks), and 